\title{
DOENÇA HEPÁTICA NÃO-ALCOÓLICA: evolução após derivação gastrojejunal em Y-de-Roux pela técnica de Fobi-Capella
}

\author{
Alexandre Coutinho Teixeira de FREITAS ${ }^{1,2}$, Diane Teixeira de FREITAS ${ }^{3}$, \\ Mônica Beatriz PAROLIN ${ }^{1}$, Antônio Carlos Ligocki CAMPOS ${ }^{1,2}$ e Júlio Cezar Uili COELHO ${ }^{1,2}$
}

\begin{abstract}
RESUMO - Racional - A doença hepática não-alcoólica apresenta alta prevalência entre pacientes com obesidade mórbida, podendo evoluir de esteatose hepática até esteatohepatite e cirrose. Objetivo - Determinar o efeito da derivação gástrica na incidência de doença hepática nãoalcoólica e co-morbidades relacionadas em pacientes com obesidade mórbida. Métodos - Os pacientes foram prospectivamente avaliados no pré-operatório e, no mínimo, após 6 meses de pós-operatório. Foram analisados: dados antropométricos, co-morbidades, medicamentos em uso, colesterol, triglicerídeos, provas hepáticas e incidência de doença hepática não-alcoólica. Todos os pacientes com alteração de provas hepáticas foram submetidos a biopsia hepática no peroperatório. Resultado - Vinte e oito pacientes com doença hepática não-alcoólica foram incluídos no estudo com índice de massa corpórea médio de $42 \pm 4 \mathrm{~kg} / \mathrm{m}^{2}$. Vinte e cinco pacientes apresentaram 59 co-morbidades, sendo as mais freqüentes: elevação de triglicerídeos $(n=23)$, elevação de colesterol total $(n=13)$ e hipertensão arterial $(n=11)$. Foram submetidos a biopsia 22 pacientes: 10 apresentaram esteatose macrogoticular moderada, 5 esteatose macrogoticular discreta e 7 esteatohepatite. Os doentes foram analisados em média após 230 dias de pós-operatório. Apresentaram perda de $64 \%$ do excesso de peso, redução do índice de massa corpórea médio para $29,6 \pm 3 \mathrm{~kg} / \mathrm{m}^{2}$ e 21 co-morbidades em 13 pacientes. Houve diminuição estatisticamente significante do número dos acometidos de elevação de triglicerídeos, elevação de colesterol total, hipertensão arterial e na incidência de doença hepática não-alcoólica. Conclusão - A perda de peso proporcionada pela derivação gastrojejunal em Y-de-Roux pela técnica de Fobi-Capella em pacientes com doença hepática não-alcoólica está associada à diminuição de sua incidência e de outras co-morbidades.
\end{abstract}

DESCRITORES - Hepatopatias. Fígado gorduroso. Obesidade mórbida. Derivação gástrica. Anastomose em Y-de-Roux.

\section{INTRODUÇÃO}

A doença hepática não-alcoólica apresenta alta prevalência na população ${ }^{(9,16)}$. Ela consiste de várias situações, desde a esteatose hepática e esteatohepatite, até a cirrose e hepatocarcinoma ${ }^{(20,32)}$. Atualmente existem evidências de se considerá-la como processo evolutivo ${ }^{(20)}$. A diabetes tipo 2 e a dislipidemia são considerados fatores de risco para a doença ${ }^{(21,23)}$. No entanto, o fator mais importante é a presença de obesidade ${ }^{(22,32)}$. Esses fatores, associados à hipertensão arterial, compõem a síndrome metabólica, observada habitualmente na doença hepática não-alcoólica primária ${ }^{(23)}$. A doença hepática não-alcoólica secundária ocorre como conseqüência de cirurgias de derivação intestinal, rápida perda de peso, nutrição parenteral total, medicamentos (amiodarona), lipodistrofia e doença de Wilson ${ }^{(23)}$.

Nos Estados Unidos, de acordo com o National Health and Nutrition Examination Survey III, a prevalência de doença hepática não-alcoólica primária aumenta de acordo com o índice de massa corporal $\mathrm{IMC}^{(16)}$. Outros estudos determinam a prevalência de doença hepática não-alcoólica em pacientes com indicação de cirurgia bariátrica. MARCEAU et al. ${ }^{(19)}$ demonstraram prevalência de $86 \%$ para esteatose, $23 \%$ para esteatohepatite e $2 \%$ para cirrose em 551 pacientes.

O desenvolvimento de esteatose hepática é o primeiro processo na fisiopatologia da doença. Pacientes obesos apresentam resistência periférica à insulina e níveis séricos desse hormônio elevados. Isso determina aumento do transporte 
de ácidos graxos do tecido adiposo para o figado desenvolvendo a esteatose - denominado "primeiro impacto" $(10,11)$. Nessa fase o fígado encontra-se muito suscetível a insultos adicionais como consumo de etanol e lipopolissacarídeos bacterianos e a apoptose torna-se fenômeno mais freqüentemente observado ${ }^{(30,42)}$. A progressão para esteatohepatite e cirrose não é totalmente esclarecida. No entanto, o estresse oxidativo e as citocinas são os principais efetores dessa evolução - chamado "segundo impacto" $(4,10,18,31,37,40)$. Indivíduos obesos apresentam alta prevalência de apnéia do sono. A hipoxemia intermitente secundária à apnéia pode participar na fisiopatologia da doença hepática não-alcoólica interferindo com o estresse oxidativo e resistência à insulina ${ }^{(6,29,35)}$. Também deve-se considerar o consumo aumentado de gordura e carboidratos na dieta, que também interferem no mesmo mecanismo ${ }^{(29,35)}$.

O presente estudo teve como objetivo avaliar o efeito da derivação gástrica em Y-de-Roux pela técnica de Fobi-Capella na incidência de doença hepática não-alcoólica e co-morbidades relacionadas em pacientes com obesidade mórbida.

\section{MÉTODOS}

No período de $1^{\circ}$ de fevereiro de 2003 até $1^{\circ}$ fevereiro de 2004 foram prospectivamente analisados pacientes com obesidade mórbida atendidos na clínica privada de um dos pesquisadores. Aqueles com indicação cirúrgica, operados e com esteatose hepática foram incluídos no estudo. A indicação cirúrgica foi realizada de acordo com os mesmos critérios atualmente estabelecidos pelo Conselho Federal de Medicina: a) IMC $\geq 40 \mathrm{~kg} / \mathrm{m}^{2}$ ou IMC entre 35 e $39,9 \mathrm{~kg} / \mathrm{m}^{2}$ associado à co-morbidade relacionada à obesidade; b) obesidade refratária a tratamento clínico conservador.

No pré-operatório, os pacientes foram avaliados conforme protocolo padronizado de exames complementares e consultas com especialidades. De interesse para o estudo determinou-se: idade, sexo, peso corporal, IMC, freqüência das co-morbidades (incluindo-se história de hepatite B e C), medicações em uso, hábitos etílicos. Também, resultados de exames complementares: AST, ALT, triglicerídeos, colesterol total, saturação da transferrina e ultra-sonografia abdominal. O diagnóstico das co-morbidades foi determinado baseado no relato do paciente associado à análise dos medicamentos em uso ou através da detecção nos exames pré-operatórios.

Pacientes com alteração de qualquer uma das provas hepáticas foram submetidos a biopsia hepática peroperatória. A cirurgia realizada foi a derivação gastrojejunal em Y-de-Roux pela técnica de Fobi-Capella, por via laparotômica.

No pré-operatório, o diagnóstico de esteatose hepática foi realizado de três maneiras: a) achado da ultra-sonografia préoperatória isoladamente; b) critério de Clark $^{(16)}$ - AST $>37$ U/L ou ALT $>40 \mathrm{U} / \mathrm{L}$ (sexo masculino); AST ou ALT>31 U/L (sexo feminino); ambas as situações na ausência de consumo etílico importante, hepatite $\mathrm{B}$ e C e saturação da transferrina $>50 \%$; c) associação das duas situações anteriores.

Após no mínimo 6 meses de pós-operatório, analisou-se: peso, percentagem de perda do excesso de peso, IMC, freqüência das co-morbidades, medicações em uso, os mesmos exames laboratoriais e incidência de esteatose. A percentagem de perda do excesso de peso ( $\%$ PEP) foi calculada pela fórmula: $\% \mathrm{PEP}=$ (peso inicial - peso atual / peso inicial - peso ideal) x100. O peso atual foi considerado como aquele no momento da avaliação no pós-operatório. O peso ideal foi baseado no estudo populacional da Metropolitan Life Insurance Company.

Os valores do peso, IMC, colesterol total e triglicerídeos foram analisados pelo teste $t$. A freqüência de ocorrência da esteatose e a freqüência de ocorrência de todas as outras co-morbidades, medicações em uso e freqüência de alteração de provas hepáticas foram analisadas pelo teste exato de Fisher. Considerou-se o nível de significância de $0,05(P \leq 0,05)$.

\section{RESULTADOS}

No período de $1^{\circ}$ de fevereiro de 2003 até $1^{\circ}$ fevereiro de 2004 foram atendidos 50 pacientes com obesidade mórbida com indicação cirúrgica. Desses, 28 (56\%) foram diagnosticados com esteatose hepática e incluídos no estudo (Tabela 1). Não houve mortalidade precoce ou tardia no pós-operatório. Os pacientes foram analisados em média com $230 \pm 75$ dias de pós-operatório. A $\%$ PEP foi em média $64,3 \% \pm 11 \%$. No pós-operatório houve redução significativa do peso médio $(P<0,01)$ e do IMC médio $(P<0,01)$ (Tabela 2).

TABELA 1. Sexo e idade média dos pacientes avaliados

\begin{tabular}{lcc}
\hline Sexo & $\mathbf{n}$ & Média idade \pm DP \\
\hline Masculino & 10 & $36,7 \pm 11$ anos \\
Feminino & 18 & $38,2 \pm 9$ anos \\
Total & 28 & $37 \pm 10$ anos \\
\hline
\end{tabular}

$\mathrm{DP}=$ desvio padrão

TABELA 2. Peso médio e IMC médio dos pacientes no pré e pósoperatório

\begin{tabular}{lccc}
\hline & Pré-operatório & Pós-operatório & $\boldsymbol{P}$ \\
\hline Peso médio $\pm \mathrm{DP}$ & $115,4 \pm 15 \mathrm{~kg}$ & $81,9 \pm 11 \mathrm{~kg}$ & $<0,01$ \\
$\mathrm{IMC}$ médio $\pm \mathrm{DP}$ & $42,0 \pm 4 \mathrm{~kg} / \mathrm{m}^{2}$ & $29,6 \pm 3 \mathrm{~kg} / \mathrm{m}^{2}$ & $<0,01$ \\
\hline
\end{tabular}

$\mathrm{DP}=$ desvio padrão

IMC = índice de massa corpórea

Os pacientes apresentaram no pré-operatório 59 comorbidades (exceto a esteatose hepática) - média de 2,07 por paciente (Tabela 3). Não foi detectado hábito etílico significativo, diagnóstico de hepatite viral ou saturação da transferrina $>50 \%$. No pós-operatório, apresentaram 21 co-morbidades, média de 0,75 por paciente, incluindo-se 6 co-morbidades relacionadas à cirurgia, ausentes no pré-operatório (Tabela 3 ). Co-morbidades foram evidenciadas em 25 pacientes no pré-operatório e em 13 pacientes no pós-operatório $(P<0,01)$ (Tabela 3$)$. Houve redução significativa das taxas de triglicerídeos $(P<0,01)$ e colesterol $(P=0,01)$ e da freqüência de ocorrência de hipertensão arterial $(P<0,05)$ (Tabela 3$)$. No pré-operatório, 15 pacientes usavam algum tipo de medicamento; no pós-operatório, $8(P=0,1)$. Todos os pacientes usaram suplementação com complexo vitamínico 
TABELA 3. Co-morbidades presentes no pré e pós-operatório

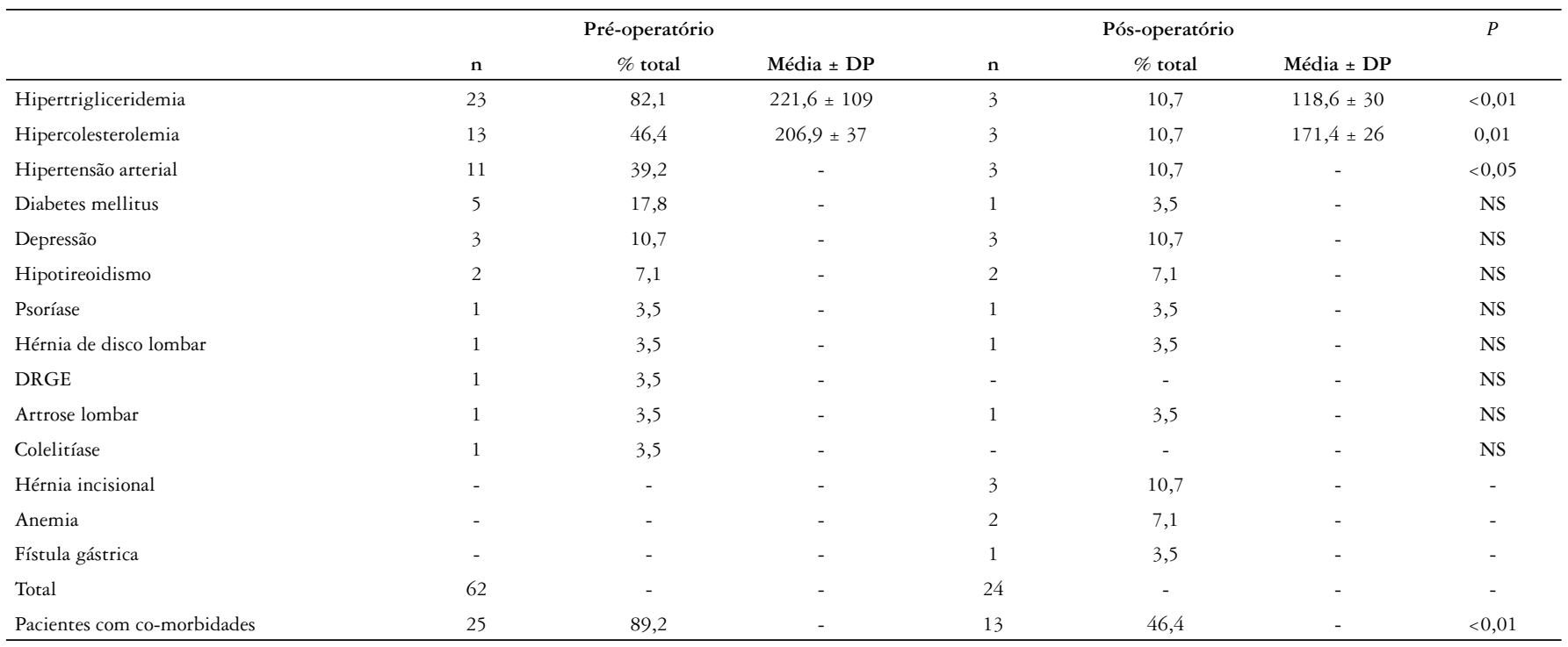

DRGE = doença do refluxo gastroesofágico

no pós-operatório, o que não foi computado. Os medicamentos usados no pré e no pós-operatório estão listados na Tabela 4.

Em 50\% dos casos o diagnóstico da esteatose hepática foi realizado com a associação da ultra-sonografia pré-operatória e alteração das provas hepáticas do critério de Clark (Tabela 5). Ao todo, 22 pacientes foram submetidos a biopsia hepática. Os resultados estão listados na Tabela 6 . Houve redução da incidência de AST $(P<0,05)$ e ALT $(P<0,01)$ elevadas no pós-operatório em relação ao pré-operatório. Vinte pacientes apresentaram elevação de ALT, AST ou ambas. Desses, em $95 \%$ dos casos a ALT estava mais elevada. A relação ALT/AST foi de 1,5. No pós-operatório houve redução significativa na incidência de esteatose hepática $(P<0,01)$, sendo observada em 11 pacientes. O diagnóstico foi realizado através de ultra-sonografia e ALT elevada em um caso, elevação isolada de AST em quatro e elevação isolada de ALT em seis casos.

\section{DISCUSSÃO}

A obesidade é considerada o fator de risco mais importante no desenvolvimento de doença hepática não-alcoólica. Acredita-se que a prevalência da doença esteja aumentando devido ao comprovado aumento da obesidade em diversos países ocidentais ${ }^{(16)}$. Poucos estudos determinam a prevalência da doença hepática não-alcoólica em obesos. Ainda, os resultados desses estudos variam de acordo com o método utilizado para o diagnóstico ${ }^{(16)}$.

Em estudo de pacientes com obesidade no norte da Itália, a prevalência de doença hepática não-alcoólica foi de $76 \% \%^{(3)}$. Nesse estudo, o método usado para o diagnóstico foi a ultra-sonografia. Normalmente observa-se fígado esbranquiçado, brilhante, se comparado com a ecogenicidade renal ${ }^{(12,17)}$. Apresenta ainda sombras acústicas posteriores e perda da definição da imagem dos vasos intrahepáticos $^{(12,17)}$. A sensibilidade varia de $67 \%$ a $100 \%$ e a especificidade de $77 \%$ a 95\% para a detecção de esteatose ${ }^{(8,17,32,34)}$. Para a detecção
TABELA 4. Medicações usadas pelos pacientes no pré e no pósoperatório

\begin{tabular}{lcc}
\hline Medicação & Pré-operatório & Pós-operatório \\
\hline Inibidor ECA & 4 & - \\
$\beta$ bloqueador & 3 & 1 \\
Antidepressivo & 3 & 3 \\
Hipoglicemiante oral & 4 & 1 \\
Diurético & 3 & 1 \\
Hipolipemiante & 2 & 1 \\
Hormônio tireóideo & 2 & 2 \\
Antianêmico & - & 2 \\
Antagonista de canal cálcio & 1 & - \\
Insulina & 1 & - \\
Antimetabólito & 1 & - \\
Total & 24 & 11 \\
\hline
\end{tabular}

TABELA 5. Diagnóstico da esteatose hepática

\begin{tabular}{lcc}
\hline Diagnóstico esteatose & n & $\%$ \\
\hline Achado ultra-sonografia + PH & 14 & 50 \\
Achado ultra-sonografia & 8 & 28,6 \\
PH & 6 & 21,4 \\
\hline
\end{tabular}

$\mathrm{PH}=$ alteração de provas hepáticas do critério de Clark

TABELA 6. Alterações nas biopsias hepáticas obtidas durante a cirurgia

\begin{tabular}{lcc}
\hline Alteração & $\mathbf{n}$ & $\%$ \\
\hline Esteatose macrogoticular moderada & 10 & 45,4 \\
Esteatose macrogoticular discreta & 5 & 22,7 \\
Esteatohepatite + esteatose macrogoticular severa & 2 & 9,1 \\
Esteatohepatite + esteatose macrogoticular moderada & 2 & 9,1 \\
Esteatohepatite + esteatose macrogoticular discreta & 2 & 9,1 \\
Esteatohepatite com fibrose portal & 1 & 4,6 \\
Total & 22 & 100 \\
\hline
\end{tabular}


de fibrose a sensibilidade varia de $57 \%$ a $77 \%$ e a especificidade de $85 \%$ a $89 \%{ }^{(8,17,32,34)}$. Trata-se, atualmente, do método de imagem de escolha para o diagnóstico visto sua praticidade, disponibilidade e custo. A associação de achados ecográficos com alteração de transaminases confere valor preditivo positivo de $96 \%$ para o diagnóstico de doença hepática não-alcoólica ${ }^{(17)}$.

Os níveis de AST e ALT podem ser utilizados para o diagnóstico ${ }^{(44)}$ Em $65 \%$ a $95 \%$ dos doentes com doença hepática não-alcoólica, a ALT é mais elevada que a AST ${ }^{(44)}$. Em contraste, em indivíduos com doença hepática alcoólica habitualmente a AST é mais elevada que a $\mathrm{ALT}^{(24,32)}$. Outras enzimas hepáticas como a fosfatase alcalina e a gama-glutamil-transferase estão elevadas em 1/3 dos pacientes. Normalmente não se observam alterações de albumina, coagulação e bilirrubinas, exceto quando a cirrose já está presente ${ }^{(12)}$. Os dados do National Health and Nutrition Examination Survey III (NHANES) analisaram a prevalência de doença hepática não-alcoólica na população adulta dos Estados Unidos com três diferentes critérios ${ }^{(16)}$. O critério modificado de Ruhl considerou o diagnóstico de doença hepática não-alcoólica no caso de ALT $>40$ $\mathrm{U} / \mathrm{L}$ na ausência de hepatite $\mathrm{B}$ ou $\mathrm{C}$, consumo significativo de álcool e saturação da transferrina $>50 \%$. O diagnóstico foi positivo em $6,6 \%$ dos pacientes com IMC de $30-34,9 \mathrm{~kg} / \mathrm{m}^{2}$ e em $6,8 \%$ dos casos com IMC $\geq 35 \mathrm{~kg} / \mathrm{m}^{2}$. O critério italiano considerou o diagnóstico nos indivíduos do sexo masculino com ALT $>30 \mathrm{U} / \mathrm{L}$ e nos do sexo feminino com ALT $>19 \mathrm{U} / \mathrm{L}$ na ausência de hepatite $\mathrm{B}$ ou $\mathrm{C}$, consumo significativo de álcool e saturação da transferrina $>50 \%$. O diagnóstico foi positivo em $18,1 \%$ dos pacientes com IMC de $30-34,9 \mathrm{~kg} / \mathrm{m}^{2}$ e em $24 \%$ dos com IMC $\geq 35 \mathrm{~kg} / \mathrm{m}^{2}$. O critério de Clark considerou o diagnóstico nos indivíduos do sexo masculino com AST $>37 \mathrm{U} / \mathrm{L}$ ou ALT $>40 \mathrm{U} / \mathrm{L}$ e nos do sexo feminino com AST ou ALT $>31 \mathrm{U} / \mathrm{L}$ na ausência de hepatite $\mathrm{B}$ ou C, consumo significativo de álcool e saturação da transferrina $>50 \%$. O diagnóstico foi positivo em 9,3\% dos pacientes com IMC de $30-34,9 \mathrm{~kg} / \mathrm{m}^{2} \mathrm{e}$ em $11,6 \%$ dos com IMC $\geq 35 \mathrm{~kg} / \mathrm{m}^{2}$.

No presente estudo utilizou-se como critério diagnóstico a associação do critério de Clark com a ultra-sonografia. O diagnóstico de esteatohepatite foi realizado somente através de biopsia hepática, quando indicada. No seguimento dos casos utilizaram-se os mesmos critérios.

DIXON et al. ${ }^{(13)}$ realizaram biopsia hepática 25,6 meses após a colocação de banda gástrica laparoscópica e observaram diminuição da esteatose, da atividade inflamatória e do grau de fibrose centrolobular.

A síndrome metabólica, ou síndrome X, é causada pela resistência periférica à insulina ${ }^{(7,27)}$ relacionada à ingestão de grandes quantidades de carboidratos e gordura saturada, resultando no aumento dos ácidos graxos plasmáticos e depósito de tecido adiposo ${ }^{(7,27)}$. Obesidade é observada em $40 \%$ a $100 \%$ dos pacientes, $20 \%$ a $75 \%$ apresentam diabetes mellitus tipo 2 e $20 \%$ a $81 \%$, hiperlipidemia ${ }^{(7,27)}$. A doença hepática não-alcoólica está presente em proporção elevada de pacientes e o foco principal de seu tratamento é a mudança de hábitos de vida e, principalmente, perda de peso. Perda de $10 \%$ do peso já é suficiente para melhora das transaminases e de outros fatores relacionados à síndrome metabólica ${ }^{(1,39,41,43)}$. Normalmente observa-se melhora da esteatose e esteatohepatite, no entanto em somente $50 \%$ dos pacientes há melhora do grau de fibrose ${ }^{(43)}$.
Existe preocupação na literatura médica a respeito dos efeitos deletérios para o fígado relacionados a grandes perdas de peso em curto período de tempo. Existem referências a não se exceder perda de $1 \mathrm{~kg}$ por semana ${ }^{(1)}$. SLIGTER et al. ${ }^{(38)}$ descreveram um paciente com esteatose hepática submetido a derivação gástrica em Y-de-Roux com redução do IMC de $52 \mathrm{~kg} / \mathrm{m}^{2}$ para $24 \mathrm{~kg} / \mathrm{m}^{2}$ e perda de $30 \mathrm{~kg}$ em 1 ano. Houve piora dos níveis de transaminases e a biopsia hepática no pós-operatório demonstrou esteatohepatite e fibrose hepática graus 1 e 2.

Outro fator importante é a realização de exercícios físicos. Foram observados benefícios dos exercícios, mesmo na ausência de perda de peso. Ocorre melhora da sensibilidade à insulina, decréscimo dos depósitos de gordura visceral, melhor utilização de ácidos graxos pelos músculos e melhora da atividade da lipase $^{(15)}$. A capacidade de realização de exercícios físicos no pós-operatório de cirurgia bariátrica é melhorada. $\mathrm{O}$ método mais usado para análise pós-operatória é o sistema BAROS - Bariatric Analysis and Reporting Outcome System ${ }^{(28)}$. Nesse método, além da perda de peso e melhora das co-morbidades, verificam-se cinco aspectos relacionados à qualidade de vida, entre eles a realização de exercícios físicos. Vários autores demonstram melhora na pontuação do sistema BAROS após a cirurgia bariátrica, refletindo, entre outros fatores, em maior capacidade física e realização de exercícios ${ }^{(14,25,36,37)}$.

Fatores dietéticos também são importantes e estão implicados na patogênese da doença hepática não-alcoólica ${ }^{(5,26)}$. No seu tratamento os pacientes são orientados a realizar dieta pobre em gordura saturada, pobre em sal, sem consumo de bebidas alcoólicas e rica em fibras, vegetais e frutas ${ }^{(2,33)}$. No pré e no pós-operatório de cirurgia bariátrica todos os pacientes são submetidos a avaliação e orientação nutricional. No presente estudo os pacientes foram acompanhados por nutricionista e orientados a modificar seus hábitos dietéticos, de acordo com a restrição imposta pela cirurgia. A derivação gástrica em Y-deRoux é considerada cirurgia mista em relação ao mecanismo de perda de peso. Nessa situação predomina mais o componente restritivo da redução do volume gástrico do que o componente disabsortivo proporcionado pelo Y-de-Roux. O volume gástrico no pós-operatório imediato é, em média, de $30 \mathrm{~mL}$. Isso torna necessária uma dieta hipocalórica fracionada e em pequenos volumes. No decorrer do tempo o volume é gradualmente aumentado. Síndrome de dumping é freqüente em pacientes que não seguem a dieta apropriadamente. A derivação gástrica permite que o paciente apresente saciedade precoce e mesmo redução do apetite, fatores mediados por alterações neurohormonais.

Neste estudo houve significativa perda de peso com a derivação gastrojejunal em Y-de-Roux, associada à redução na incidência de esteatose hepática e de outras co-morbidades. O seguimento médio foi de $230 \pm 75$ dias. Diversos estudos demonstram que nesse período ainda não ocorreu a perda de peso máxima possível com o procedimento. Especulou-se que com períodos de seguimento mais prolongados obter-se-ia maior perda de peso e, possivelmente, maior redução da incidência de esteatose e outras co-morbidades.

Conclui-se que ocorre redução da incidência de doença hepática não-alcoólica e suas co-morbidades em pacientes submetidos a derivação gastrojejunal em Y-de-Roux. 
Freitas ACT, Freitas DT, Parolin MB, Campos ACL, Coelho JCU. Nonalcoholic fatty liver disease: evolution after gastric bypass. Arq Gastroenterol. 2007;44(1):49-53.

ABSTRACT - Background - Nonalcoholic fatty liver disease is highly prevalent among morbidly obese patients and can progress from steatosis to steatohepatitis and chronic liver disease. Aim - To determine the effect of gastric bypass operation in the incidence of fatty liver disease and associated co-morbidities in morbidly obese patients. Methods - Patients were prospectively evaluated in the pre-operative period and after at least 6 months after operation. We analysed: antropometric data, comorbidities, use of medications, cholesterol and triglycerides levels, liver tests and incidence of nonalcoholic fatty liver disease. All patients with abnormal liver tests were subjected to per-operative liver biopsy. Results - Twenty eight patients with nonalcoholic fatty liver disease with a mean body mass index of $42 \pm 4 \mathrm{~kg} / \mathrm{m}^{2}$ were evaluated. Twenty five patients had 59 co-morbidities and the most frequent were: elevated triglycerides $(n=23)$, elevated cholesterol $(n=13)$ and elevated blood pressure $(\mathrm{n}=11)$. Biopsy was done in 22 patients: 10 presented moderate steatosis, 5 mild steatosis and 7 steatohepatitis. After follow-up of 230 days in average they presented weight excess loss of $64 \%$, body mass index reduction to $29,6 \pm 3 \mathrm{~kg} / \mathrm{m}^{2}$ and 21 co-morbidities in 13 patients. There was a significant decrease in the number of patients with elevated triglycerides, elevated cholesterol, elevated blood pressure and in the incidence of nonalcoholic fatty liver disease. Conclusion - The weight loss secondary to the gastric bypass is associated with decrease in the incidence of nonalcoholic fatty liver disease and other co-morbidities.

HEADINGS - Liver diseases. Fatty liver. Obesity, morbid. Gastric bypass. Anastomosis, Roux-en-Y.

\section{REFERÊNCIAS}

1. Angulo P, Lindor KD. Treatment of nonalcoholic steatohepatitis. Best practice and research. Clin Gastroenterol. 2002;16:797-810.

2. Astrup A, Ryan L, Grunwald GK, Storgaard M, Saris W, Melanson E. The role of dietary fat in body fatness: evidence from a preliminary meta-analysis of ad libitum low-fat dietary intervention studies. Br J Nutr. 2000;83:s25-s32.

3. Bellentani S, Saccoccio G, Masutti F, Croce LS, Brandi G, Sasso F. Prevalence and risk factors for hepatic steatosis in Northern Italy. Ann Intern Med. 2000;132:112-7.

4. Berson A, Beco VD, Letteron P, Robin M, Moreau C, Kahwaji JE, Verthier N, Feldmann G, Fromenty B, Pessayre D. Steatohepatits-inducing drugs cause mitochondrial dysfunction and lipid peroxidation in rat hepatocytes. Gastroenterology. 1998;114:764-74.

5. Cassader M, Gambino R, Musso G, Depetris N, Mecca F, Cavallo-Perin P, Pacini G, Rizzetto M, Pagano G. Postprandial trygliceride-rich lipoprotein metabolism and insulin sensitivity in nonalcoholic steatohepatitis patients. Lipids. 2001;36:1117-24.

6. Chalassani N, Gorski JC, Asghar MS, Ali A, Forseman B, Hall SD. Hepatic cytochrom P450 2E1 activity in non-diabetic patients with non-alcoholic steatohepatitis. Hepatology. 2003;37:544-50

7. Chitturi S, Abeygunasekera S, Farrel GC, Holmes WJ, Hui JM, Fung C. NASH and insulin resistance: insulin hypersecretion and specific association with the insulin resistance syndrome. Hepatology. 2002;35:373-9.

8. Clark JM, Brancati FL, Diehl AM. Nonalcoholic fatty liver disease. Gastroenterology 2002;122:1649-57

9. Cotrim HP, Pereira LM, Mattos AA. Doença hepática gordurosa não-alcoólica (DHGNA): inquérito com membros da Sociedade Brasileira de Hepatologia. GED Gastroenterol Endosc Dig. 2004;23;107-12.

10. Day CP, James O. Steatohepatitis: a tale of two hits? Gastroenterology. 1998;114:842-5.

11. Day CP. Nonalcoholic steatohepatitis (NASH): where are we now and where are we going? Gut. 2002;50:585-8.

12. De Knegt RJ. Nonalcoholic steatohepatitis: clinical significance and pathogenesis Scand J Gastroenterol. 2001;234:88-92

13. Dixon JB, Bhathal OS, Hughes NR, O'Brien PE. Nonalcoholic fatty liver disease: improvement in liver histological analysis with weight loss. Hepatology. 2004;39:1647-54.

14. Faintuch J, Rudner MA, Machado PLRC, Garrido AB, Oliveira MR, Gama-Rodrigue JJ. Response of comorbidities to Roux-em-Y gastric bypass (RYGBP). Obes Surg. 2001:11:430.

15. Hardman AE. Physical activity, obesity and blood lipids. Int J Obes Relat Metab Disord. 1999;23:s64-s71.

16. Haynes $\mathrm{P}$, Liangpunsakul $\mathrm{S}$, Chalasani $\mathrm{N}$. Nonalcoholic fatty liver disease in individuals with severe obesity. Clin Liver Dis. 2004;8:535-7.

17. Joy D, Thava VR, Scott BB. Diagnosis of fatty liver disease: is biopsy necessary? Eur J Gastroenerol Hepatol. 2003;15:539-43.

18. Kern PA, Saghizadeh M, Ong J, Bosh R, Deem R, Simsolo R. The expression of tumor necrosis factor in human adipose tissue: regulation by obesity, weight loss, and relationship to lipoprotein lipase. J Clin Invest. 1995;95:2111-9.

19. Marceau P, Biron S, Hould FS, Marceau S, Simard S, Thung SN. Liver pathology and the metabolic syndrome X in severe obesity. J Clin Endocrinol Metab. 1999;84:1513-7.

20. Matteoni CA, Younossi ZM, Gramlich T, Boparai N, Liu YC, McCullough AJ. Nonalcoholic fatty liver disease: a spectrum of clinical and pathological severity. Gastroenterology. 1999;116:1413-9.

21. Mattos AA. Esteato-hepatite não-alcoólica. J Bras Gastroenterol. 2005;5:160-5.

22. McCullough AJ. Update on nonalcoholic fatty liver disease. J Clin Gastroenterol. $2002 \cdot 34 \cdot 255-62$
23. Medina J, Garcia-Buey L, Fernandez-Salazar LI, Moreno-Otero R. Approach to the pathogenesis and treatment of nonalcoholic steatohepatitis. Diabetes Care. 2004;27:2057-66.

24. Mehta K, Van Thiel DH, Shah N, Mobarhan S. Nonalcoholic fatty liver disease: pathogenesis and the role of antioxidants. Nutr Rev. 2002;60:289-93.

25. Moorehead MK, Ardelt-Gattinger E, Lechner H, Oria H. The validation of the Moorehead-Ardelt quality of life questionnaire II. Obes Surg. 2003;13:684-92.

26. Musso G, Gambino R, De Michielli F, Cassader M, Rizzeto M, Durazzo M. Dietary habits and their relations to insulin resistance and postprandial lipemia in nonalcoholic steatohepatitis. Hepatology. 2003;37:909-16.

27. Neuschwander TBA, Caldwell SH. Nonalcoholis steatohepatitis: summary of an AASLD Single Topic Conference. Hepatology. 2003;37:1202-19.

28. Oria HE, Moorehead MK. Bariatric analysis and reporting outcome system (BAROS). Obes Surg. 1999;8:487-99.

29. Punjabi NM, Ahmed MM, Polotsky VY, Beamer BA, O'Donnel CP. Sleep-disordered breathing, glucose intolerance, and insulin resistance. Respir Physiol Neurobiol. 2003;136:167-78

30. Rashid A, Wu T, Huang C, Chen C, Lin H, Yang S, Lee F, Diehl A. Mitochondrial proteins that regulate apoptosis and necrosis are induced in mouse fatty liver. Hepatology. 1999;29:1131-8.

31. Reddy JK. Nonalcoholic steatosis and steatohepatitis. III. Peroxisomal beta-oxidation, PPAR alpha and steatohepatitis. Am J Physiol Gastrointest Liver Physiol. 2001;281: g1333-g9.

32. Reid AE. Nonalcoholic steatohepatitis. Gastroenterology. 2001;121:710-23.

33. Riccardi G, Rivellese AA. Dietary treatment of the metabolic syndrome - the optimal diet. Br J Nutr. 2000;83:s143-s8.

34. Saadeh S, Younossi ZM, Remer EM, Gramlich T, Ong JP, Hurley M. The utility of radiological imaging in nonalcoholic fatty liver disease. Gastroenterology. 2002;123:745-50.

35. Schafer H, Pauleit D, Sudhop T, Gouni-Berthold I, Ewig S, Berthold HK. Body fat distribution, serum leptin, and cardiovascular risk factors in men with obstructive sleep apnea. Chest. 2002;122:829-39.

36. Schauer PR, Ikramuddin S, Gourash W, Ramanathan R, Luketich J. Outcomes after laparoscopic Roux-en-Y gastric bypass for morbid obesity. Ann Surg. 2000;232:515-29.

37. Schoepel KL, Olchowski SE, Mathis MW, Pridgen D, Maxwell JG. Starting a successful bariatric surgical practice in the community hospital setting. Obes Surg. 2001;11:559-64.

38. Sligter K, Bourass I, Sels JP, Driessen A, Stockbrugger RW, Koek GH. Non-alcoholic steatohepatitis: review of a growing medical problem. Eur J Intern Med. 2004;15:10-21.

39. Stone NJ, Krushner R. Effect of dietary modification and treatment of obesity. Emphasis on improving vascular outcome. Med Clin North Am. 2000;84:95-122.

40. Tilg H, Diehl A. Cytokines in alcoholic and nonalcoholic steatohepatitis. N Engl J Med. 2000;343:1467-76

41. Trembley A. Physical activity and obesity Bailliere's best practice and research. Clin Endocrinol Metab. 1999;13:121-9.

42. Unger RH, Orci L. Lipoapoptosis: its mechanism and its diseases. Biochem Biophis Acta. 2002;1585:202-12

43. Youssef WI, McCullough AJ. Steatohepatitis in obese individuals. Best practice and research. Clin Gastroenterol. 2002;16:733-47.

44. Zamin I, Mattos AA, Perin C, Ramos GZ. A importância do índice AST/ALT no diagnóstico da esteatohepatite não-alcoólica. Arq Gastroenterol. 2002;39:22-6. 